\title{
Importancia de la vitamina B12 y el folato en la salud perinatal
}

Ameyalli M. Rodríguez-Cano1, Otilia Perichart-Perera ${ }^{1 *}$ y Pedro Gutiérrez-Castrellón ${ }^{2}$

${ }^{1}$ Departamento de Nutrición y Bioprogramación, Instituto Nacional de Perinatología Isidro Espinosa de los Reyes; ${ }^{2}$ Centro de Investigación Translacional en Ciencias de la Salud, Hospital General Dr. Manuel Gea González; ${ }^{3}$ Sociedad Latinoamericana de Gastroenterologia, Hepatología y Nutrición Pediátrica. Ciudad de México, México

\section{Resumen}

El desarrollo fetal se caracteriza por una gran plasticidad y capacidad para responder a factores ambientales, donde la metilación del ADN es indispensable para el desarrollo embrionario adecuado. El metabolismo de un carbono proporciona grupos metilo para la metilación y el desarrollo del ADN fetal, y depende en gran medida del estado nutricio materno. El embarazo es una etapa donde el suministro de donantes de metilo es crítico y la demanda de nutrimentos que apoyen este proceso, como lo son el folato y la vitamina B12, está aumentada. La insuficiencia o desequilibrio de estos dos micronutrimentos puede alterar los patrones epigenéticos, la síntesis y reparación del ADN, y afectar procesos del crecimiento y desarrollo fetal, teniendo consecuencias negativas en la salud de la descendencia a largo plazo. El estado del folato y la vitamina B12 se han asociado con la metilación global del ADN, así como con genes específicos relacionados con funciones neurológicas, con el desarrollo embrionario, el metabolismo energético, el crecimiento, y con la leptina. Además, estados alterados de ambas vitaminas se han asociado con mayor riesgo de resultados perinatales como defectos del tubo neural, prematurez, bajo peso al nacer, preeclampsia, así como obesidad y resistencia a la insulina materna e infantil, y disminución del desarrollo neurocognitivo infantil. La suplementación, aunada a una dieta adecuada, podría ser una estrategia necesaria para prevenir dichos resultados y mejorar la salud maternofetal.

PALABRAS CLAVE: Dieta. Micronutrimentos. Metilación. Programación fetal.

\section{Importance of vitamin B12 and folate in perinatal health}

\begin{abstract}
Fetal development is characterized by great plasticity and the ability to respond to environmental factors, where DNA methylation is essential for proper embryonic development. One-carbon metabolism provides methyl groups for methylation and fetal DNA development and is highly dependent on maternal nutritional status. During pregnancy, the supply of methyl donors is critical and the demand for nutrients that support this process, such as folate and vitamin B12, is increased. Insufficiency or imbalance of these 2 micronutrients can alter epigenetic patterns, DNA synthesis and repair, and affect fetal growth and development, having negative long-term consequences on the offspring's health. Folate and vitamin B12 status have been associated with wide DNA methylation, as well as with specific genes related to neurological functions, embryonic development, energy metabolism, growth, and leptin. Furthermore, inadequate concentrations of both vitamins have been associated with an increased risk of perinatal outcomes such as neural tube defects, prematurity, low birth weight, pre-eclampsia, as well as maternal and infant obesity and insulin resistance, and decreased infant neurocognitive development. Supplementation, combined with a healthy diet, could be an essential strategy to prevent these results and improve maternal and fetal health.
\end{abstract}

KEYWORDS: Diet. Micronutrients. Methylation. Fetal programming.

Correspondencia:

*Otilia Perichart-Perera

E-mail: otiliaperichart@inper.gob.mx
Fecha de recepción: 04-09-2020

Fecha de aceptación: 08-11-2020 DOI: 10.24875/GMM.M20000435
Gac Med Mex. 2020;156(Supl 3): S27-S36

Disponible en PubMed

www.gacetamedicademexico.com

0016-3813/C 2020 Academia Nacional de Medicina de México, A.C. Publicado por Permanyer. Este es un artículo open access bajo la licencia CC BY-NC-ND (http://creativecommons.org/licenses/by-nc-nd/4.0/). 


\section{Introducción}

El término epigenética se refiere a las modificaciones moleculares que ocurren en el ADN y su expresión génica, sin alterar la secuencia primaria. Es la forma en que se organiza, mantiene y lee la información genética ${ }^{1,2}$. Los mecanismos epigenéticos son cada vez más reconocidos, ya que posiblemente son reguladores de la etiología y la progresión de diversas enfermedades ${ }^{3,4}$. Las modificaciones epigenéticas incluyen la metilación del ADN, siendo esta la más estudiada y posiblemente mejor caracterizada, las modificaciones de la cola de histonas, la remodelación de la cromatina y la regulación génica del ARN no codificante ${ }^{1,4,5}$. Cada uno de estos procesos puede afectar la expresión génica, aunque no son independientes entre sí.

La metilación del ADN puede desempeñar un papel regulador profundo dictando qué genes se expresarán o silenciarán en una célula, tejido u órgano ${ }^{7}$, por lo que se ha visto involucrada en el desarrollo y mantenimiento de la homeostasis y las funciones celulares ${ }^{1}$. La plasticidad celular requiere que diversos mecanismos interaccionen equilibradamente, donde los mecanismos epigenéticos tienen un papel importante ${ }^{8}$.

El desarrollo fetal se caracteriza por una gran plasticidad y capacidad para responder a factores ambientales, donde la metilación del ADN es indispensable para el desarrollo embrionario adecuado. Desde la célula única hasta la etapa de blastocisto de la embriogénesis, se producen muchos cambios en la metilación del $\mathrm{ADN}^{1}$, siendo así una etapa crítica en el establecimiento del epigenoma ${ }^{8}$. Las modificaciones epigenéticas podrían ser promovidas por diversos factores ambientales y de estilo de vida, incluidos la dieta, el ejercicio y el estrés, y pueden tener profundos efectos en el desarrollo de ovocitos y embriones, sobre el desarrollo placentario, el crecimiento fetal y la neurogénesis de las vías hipotalámicas de regulación del peso corporal, entre otros ${ }^{3}$. La nutrición dentro del útero puede afectar permanentemente la expresión de diversos genes en la descendencia y determinar su función metabólica. Estos cambios en la vida intrauterina, en su mayoría estables durante toda la vida ${ }^{9}$, programan el funcionamiento de órganos (conocido como programación fetal o del desarrollo), predisponiendo a los individuos a un fenotipo obesogénico que persiste durante la edad adulta, llevando a alteraciones metabólicas y resultados de salud negativos a largo plazo ${ }^{3,6}$.
El embarazo se asocia con mayores necesidades de energía y nutrimentos debido a los cambios fisiológicos de la madre y las demandas metabólicas del feto ${ }^{10}$. Los desequilibrios o insuficiencias en los componentes de la dieta podrían entonces afectar la metilación, la expresión génica en el útero y durante la vida temprana, lo que puede dar lugar a una disparidad fenotípica, incluida la susceptibilidad incrementada de enfermedades ${ }^{1,2}$.

\section{Metilación del ADN y metabolismo de un carbono}

El epigenoma es particularmente dinámico durante la embriogénesis debido a la extensa síntesis de ADN y al elaborado patrón de metilación del ADN requerido para el desarrollo normal del tejido². A diferencia de otros mecanismos epigenéticos, como las modificaciones transitorias de histonas, las marcas de metilación del ADN son químicamente muy estables y pueden conservarse con el tiempo, lo que explica potencialmente las consecuencias a largo plazo para la salud ${ }^{8}$.

Los genomas de los gametos están altamente metilados en comparación con las células somáticas. Poco después de la fertilización, el cigoto recién formado se somete a una desmetilación global ${ }^{1,8,11}$, seguida de un nuevo patrón de metilación del ADN, predominantemente en la etapa de blastocisto ${ }^{1,8,11}$. El entorno materno puede modificar la metilación en esta etapa, en un intento de regular el desarrollo fetal acorde a los nutrimentos disponibles y otras condiciones, de modo que la descendencia pueda estar preparada para enfrentar condiciones adversas mucho después del nacimiento ${ }^{11,12}$.

La metilación implica la adición covalente de un grupo metilo a una citosina dentro de los dinucleótidos citosina-guanidina (CpG) del ADN, convirtiéndola en 5-metilcitosina $(5 \mathrm{mC})^{6,8}$. La adición del grupo metilo es por medio del donante de metilo universal, S-adenosilmetionina (SAM). Como consecuencia, la metilación del ADN depende de la disponibilidad de grupos metilo de SAM, lo cual resalta su papel como un metabolito clave en la metilación del ADN1. La metilación del ADN es catalizada por la acción de varias metiltransferasas de ADN (DNMT), que usan zinc como cofactor ${ }^{2,4,7}$ (Fig. 1A).

La metilación de la citosina cambia la propiedad hidrofóbica del ADN e inhibe su interacción con otros activadores o supresores de la transcripción. En términos generales, la hipometilación permite «activar» el 


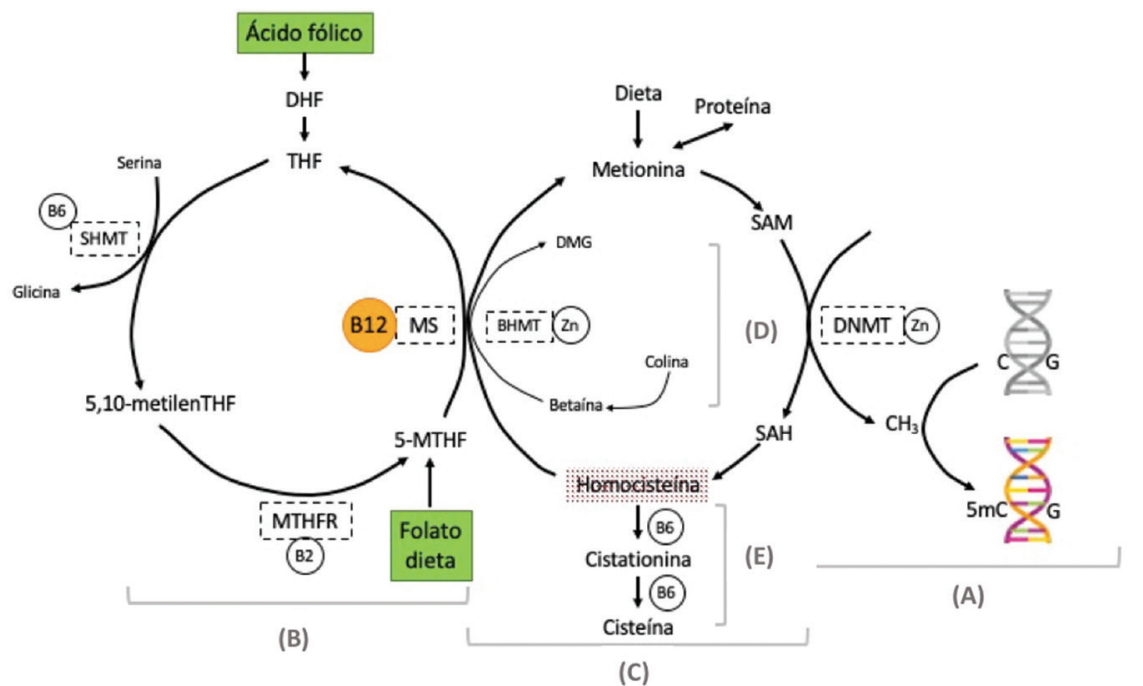

Figura 1. Folato y B12 en la metilación y ciclo de un carbono.

5mC: 5-metilcitosina; 5-MTHF: 5-metiltetrahidrofolato; BHMT: betaína homocisteína metiltransferasa; $\mathrm{CH}_{3}$ : grupo metilo; DHF: dihidrofolato; DMG: dimetilglicina; DNMT: metiltransferasa de ADN; MTHFR: metiltetrahidrofolato reductasa; MS: metionina sintasa; SAH: S-adenosil homocisteína; SAM: S-adenosil metionina; SHMT: serina hidroximetiltransferasa; THF: tetrahidrofolato; Zn: zinc.

gen y la hipermetilación lo «silencia»", sin embargo, este no es siempre el caso6,8. Los genes implicados en el metabolismo energético, la adipogénesis, la homeostasis de la glucosa, la señalización de la insulina, de leptina (LEP) y factores de transcripción adipogénicos y lipogénicos se han encontrado particularmente regulados por mecanismos epigenéticos ${ }^{9}$.

\section{Metabolismo de un carbono}

Un componente crucial del metabolismo celular es el metabolismo de un carbono (1-C), que proporciona grupos metilo (unidades de un carbono) para la metilación y el desarrollo del ADN fetal ${ }^{4,13,14}$. También proporciona grupos metilo para al menos 50 reacciones de metilación diferentes como la de ARN, ácidos nucleicos, fosfolípidos, neurotransmisores y otras proteínas. Estas reacciones sirven para la síntesis de purinas, timidilato, creatina, fosfatidilcolina y múltiples hormonas ${ }^{13,14}$.

El metabolismo de 1-C se caracteriza por una redundancia de vías para garantizar la disponibilidad del grupo metilo y evitar la acumulación de homocisteína ${ }^{7}$. Este ciclo consta de dos vías de metilación, una dependiente de folato y otra independiente de folato?.

En la vía dependiente de folato, la serina hidroximetiltransferasa (enzima que contiene vitamina B6), cataliza la transferencia de una unidad de un carbono de la serina hacia el tetrahidrofolato (THF) para generar 5,10metilenTHF; que posteriormente se reduce a 5-metilTHF (5-MTHF) por acción de la metiltetrahidrofolato reductasa (MTHFR), reacción donde interviene la vitamina B2. La 5-MTHF transfiere su unidad de carbono ahora a la metionina (aminoácido indispensable derivado de la dieta), lo cual es catalizado con ayuda de la vitamina B12 (vitB12) mediante su acción en la enzima metionina sintasa (MS) ${ }^{4,8}$. Con esta transferencia, la metionina se convierte en SAM, donde las DNMT unen los grupos metilo de SAM a la posición de carbono 5 de las bases de citosina, generando $5 \mathrm{mC}$ y metilando el $\mathrm{ADN}^{15}$ (Fig. 1B).

La S-adenosilhomocisteína (SAH) es el subproducto que queda al donar el grupo metilo de SAM. La SAH se escinde en homocisteína (Hcy) por la hidrolasa-SAH. Esta Hcy resultante se remetila para formar metionina, por acción de la MS. El grupo metilo para la remetilación de la Hcy es donado por 5-MTHF ${ }^{4,13}$ (Fig. 1C).

La vía independiente del folato utiliza la Hcy para formar metionina, pero esta vez mediante la reacción de la betaína-homocisteína metiltransferasa (que requiere zinc) usando betaína de la dieta o derivada de la colina. La metionina generada, de nuevo, se convierte en SAM para suministrar grupos metilo ${ }^{4,7}$ (Fig. 1D).

Es importante destacar que la SAH no solo es el producto final, sino que también es un inhibidor de 
las DNMT, pues tiene mayor afinidad que la SAM. Por lo tanto, la eliminación de la SAH es esencial para el flujo del ciclo de la metionina y también para la eficiencia de las reacciones de las DNMT ${ }^{4}$.

Otro mecanismo que evita la acumulación de Hcy es transformarla en cisteína (solo en hígado, riñones, páncreas, intestino y cerebro) por transulfuración irreversible, en un proceso que requiere vitamina $B 6^{3,8}$ (Fig. 1E).

El metabolismo de 1-C depende en gran medida del estado nutricio materno. La dieta proporciona moléculas donantes de metilo como la metionina, el folato y la colina, así como los cofactores, incluidos el zinc y las vitaminas B2, B6 y B12 2,7,14. El embarazo es una etapa donde el suministro de donantes de metilo es crítico y la demanda de nutrientes es mayor ${ }^{8}$. Durante el desarrollo temprano, cuando los patrones específicos del tejido están en proceso de establecimiento y maduración, el epigenoma es sensible a cambios sutiles, por lo que la insuficiencia o desequilibrio de estos micronutrientes como resultado de las deficiencias en la dieta pueden alterar los patrones epigenéticos contribuyendo a alteraciones metabólicas y degenerativas que incluso pueden presentarse a mediano y largo plazo ${ }^{3,16}$.

\section{Folato y vitamina B12 en el embarazo}

Durante el embarazo hay una mayor demanda de folato para la rápida proliferación celular, la invasión del trofoblasto y la angiogénesis para el adecuado crecimiento y desarrollo fetal, además del desarrollo saludable de la placenta y la adaptación materna al embarazo ${ }^{1,7}$. Por su importancia en la formación de la columna vertebral, el cerebro y el cráneo del feto, el ácido fólico previene defectos del tubo neural (DTN) ${ }^{7,17}$.

La ingestión diaria recomendada de folato para el embarazo es de 600-750 mcg/día, proveniente de alimentos y fuentes suplementarias ${ }^{18-20}$. El folato natural es más abundante en vegetales de hoja verde oscuro, frutas, leguminosas, chícharos, nueces, espárragos, huevo y, con excepción del hígado, la carne no es una buena fuente de ácido fólico. La forma sintética, el ácido fólico, se usa como fortificante en la harina blanca, la pasta, la harina de maíz, los cereales listos para consumir y suplementos dietéticos ${ }^{7,21}$. Un estudio encontró un consumo deficiente de folato en el $91.3 \%$ de mujeres mexicanas embarazadas $^{22}$. No hay puntos de corte universalmente aceptados para definir la deficiencia de folato durante el embarazo. Generalmente se define como un folato sérico $<7 \mathrm{nmol} / \mathrm{l}$ (indicador sensible al consumo reciente; 1-3 días previos) y un folato eritrocitario < $100 \mathrm{nmol} / \mathrm{l}$ (indicador del estado crónico: 2 a 4 meses) $)^{17,23}$. Sin embargo, el nivel de folato asociado con el riesgo más bajo de DTN es $906 \mathrm{nmol} / \mathrm{l}^{17}$.

La cobalamina (vitB12) es un compuesto necesario para la función neurológica, la síntesis de ADN y la formación de la sangre ${ }^{7,24}$. Durante el embarazo la ingestión recomendada de vitB12 es de $2.6 \mathrm{mcg} / \mathrm{dí}^{18-20}$. Alimentos con alto contenido de vitB12 incluyen básicamente productos de origen animal como carne roja, pollo, hígado, pescado, mariscos, huevo y productos lácteos; algunas macroalgas como la nori también la contienen. Por ello los vegetarianos, y más específicamente los veganos, son propensos a la deficiencia de vitB12 ${ }^{21,25}$. La deficiencia también resulta de la malabsorción y es común en mujeres embarazadas de entornos de bajos ingresos ${ }^{21,26}$. La evaluación del estado de vitB12 incluye marcadores bioquímicos y funcionales. Los bioquímicos incluyen vitB12 total (suero o plasma) y holotranscobalamina (indicador de la vitB12 biológicamente activa, que no se ve afectada por afecciones que disminuyen la proteína de unión a B12-haptocorrina, como el embarazo). Los marcadores funcionales incluyen el ácido metilmalónico (MMA) (la deficiencia de vitB12 conduce a una acumulación de MMA, pues su conversión en succinil-CoA para ser utilizado por la célula requiere vitB12 como cofactor) y la $\mathrm{Hcy}^{24,27}$. Recientemente se ha sugerido el uso potencial de $\mathrm{cB12}$, una combinación de los 4 biomarcadores (vitB-12 total, MMA, Hcy y holotranscobalamina) como un indicador del estado de vitB12, aunque aún no ha sido validado en entornos clínicos o de campo. La deficiencia de vitB12 se ha definido como una concentración sérica o plasmática < $148 \mathrm{pmol} / \mathrm{l}$ y la insuficiencia $<200 \mathrm{pmol} / /^{24}$. Algunos informes indican que la deficiencia de vitB12 es más prevalente de lo que se pensaba incluso en no vegetarianos ${ }^{21,25}$. La insuficiencia de vitB12 en embarazadas de varias partes del mundo puede llegar a ser entre el 20 y $30 \%$ en todos los trimestres ${ }^{28}$. En embarazadas en una población rural de México se reportó una prevalencia de deficiencia severa $(<74$ pmol/l) y valores marginales (< $148 \mathrm{pmol} / \mathrm{l})$ de B12 fue respectivamente del 19 y el $43 \%{ }^{29}$.

\section{Estado de folato y B12 y metilación}

La metilación del ADN se programa en el útero y es altamente susceptible a modificadores ambientales, incluyendo la dieta materna y la suplementación. 
En un metaanálisis de estudios de epigenoma completo de dos cohortes europeas ( $n=1,988$ recién nacidos) se identificaron 48 sitios $\mathrm{CpG}$ que se asociaron de manera inversa con las concentraciones de folato plasmático materno durante el embarazo. Aunque la mayoría de los genes involucrados no se han descrito en la biología del folato, incluyendo APC2, GRM8, SLC16A12, OPCML, PRPH, LHX1, KLK4 y PRSS21, algunos sí se relacionan con DTN, funciones neurológicas o varios aspectos del desarrollo embrionario ${ }^{30}$.

En la cohorte PREFORM en mujeres canadienses ( $n=368$, principalmente caucásicas y nulíparas) se observó que las más altas concentraciones de folato eritrocitario $(>2,860 \mathrm{nmol} / \mathrm{l})$ y más bajas concentraciones de vitB12 (< $167 \mathrm{pmol} / \mathrm{l})$, en etapas tempranas del embarazo, se asociaron con una disminución significativa en la metilación global del ADN de células mononucleares de cordón (media $5 \mathrm{mC}$ : $5.16 \%$; intervalo de confianza del 95\% [IC 95\%]: 4.98-5.35\%). En este mismo grupo (alto folato, baja vitB12) se observó un mayor peso al nacer en comparación con el grupo con bajas concentraciones de folato y altas de vitB12 (3,697 vs. $3,393 \mathrm{~g} ; p=0.01)$. En este estudio, las concentraciones maternas tempranas de vitB12 correlacionaron de manera positiva con el ADN de sangre de cordón ( $r: 0.25 ; p=0.002$ ). No se encontró asociación entre las concentraciones maternas de folato eritrocitario ni de sangre de cordón $\mathrm{n}^{31}$.

Se han observado algunas asociaciones entre el estado de folato materno y la metilación específica de algunos genes. En la cohorte prospectiva MANOE (Maternal Nutrition and Offspring's Epigenome) en Bélgica (114 parejas de madre e hijo), se encontró una asociación negativa entre el consumo materno de folato dietético antes del embarazo con la metilación de LEP (relacionada con el control del apetito) $(-1.233 \%$ de disminución en la metilación de LEP por $100 \mathrm{mcg}$ de aumento en el consumo de folato; IC 95\%: -2.342 a -0.125; $p=0.0298$ ) y de ácido fólico (de suplementos) y la metilación de IGF2 DMR (insulin-like growth factor 2, differentially methylated region, relacionado con el crecimiento) $(-0.706 \%$ de disminución en la metilación de IGF2 DMR por 100 mcg de aumento en el consumo de folato; IC 95\%: -1.242 a -0.107; $p=0.0101$ ) en el infante a los 6 meses de edad. También se reportó una asociación positiva entre el consumo de folato dietético antes del embarazo y la metilación de RXRA (retinoid $X$ receptor- $\alpha$, relacionado con metabolismo) $(0.685 \%$ de incremento en la metilación de RXRA por cada aumento de
100 mcg de consumo de folato; IC 95\%: 0.245 a 1.125; $\mathrm{p}=0.027)$. La metilación de DNMT1 en el lactante (6 meses de vida) se asoció negativamente con el consumo materno de folato en el 2 o trimestre $(-0.027 \%$ disminución en la metilación de DNMT1 por $100 \mathrm{mcg}$ de aumento en el consumo de folato; IC 95\%: -0.051 a -0.004; $p=0.0204$ ) y positivamente en el 3 . $^{\text {er }}$ trimestre de embarazo $(0.131 \%$ de incremento en la metilación de DNMT1 CpG3 por 100 mcg de aumento en el consumo de folato; IC 95\%: 0.016 a 0.246 ; $p=0.0256)^{32}$.

Otros estudios no han mostrado asociaciones de la metilación global y las concentraciones de folato, posiblemente debido a la variabilidad en la edad gestacional estudiada ${ }^{31}$.

\section{Estado materno de folato, vitamina B12, homocisteína y resultados perinatales}

La insuficiencia de micronutrimentos es especialmente problemática durante el embarazo, dada la necesidad de una mayor ingestión para cubrir los requerimientos aumentados ${ }^{21}$. Como se comentó, la deficiencia de folato y vitB12 puede alterar el metabolismo de 1-C, la modulación epigenética, la síntesis y reparación de ADN, y afectar procesos del crecimiento y desarrollo fetal. Por ello se considera importante evaluar el estado de vitB12, folato y homocisteína en el embarazo.

Las alteraciones en el consumo de folato y de vitB12 se reflejan en concentraciones circulantes más altas de Hcy (alta Hcy indica una baja ingestión; baja Hcy indica una alta ingestión) $)^{5,16}$. Estudios de la India han mostrado que la baja concentración de vitB12 contribuyó con un 41 y un $24 \%$ al riesgo atribuible de hiperhomocisteinemia, y con un 40 y un $12 \%$ a MMA alto a las 18 y 28 semanas de gestación, respectivamente ${ }^{33}$.

La obesidad materna también parece tener efecto sobre las concentraciones de folato y vitB12. En mujeres embarazadas del Reino Unido, las mujeres con concentraciones bajas de folato y de cobalamina presentaron los índices de masa corporal (IMC) más altos, y viceversa, aquellas con concentraciones elevadas de folato y de cobalamina presentaron un IMC más bajo. El aumento de un $1 \%$ en el IMC se asoció con una disminución del $0.6 \%$ en vitB12 y del $0.4 \%$ en folato ${ }^{34}$. En un estudio de 774 mujeres embarazadas de la India, aquellas con deficiencia de vitB12 en el embarazo presentaron mayor adiposidad, resistencia a la insulina y una mayor prevalencia de diabetes, tanto gestacional, como cinco años después. La 
incidencia de DMG en estas mujeres con deficiencia de vitB12 fue mayor a mayores concentraciones de folato ${ }^{35}$.

Los DTN representan la principal complicación asociada a deficiencia de folato, y esto ha sido ampliamente demostrado ${ }^{17}$. Los patrones anormales de metilación del ADN se consideran uno de los mecanismos más concebibles para explicar los DTN ${ }^{4}$. En un estudio multicéntrico de casos $(n=318)$ y controles $(n=702)$ en la India se reportó que las madres de fetos con DTN presentaron concentraciones de holotranscobalamina significativamente más bajas que los controles (25.4 vs. 31.7 pM, respectivamente; $\mathrm{p}<0.001$ ), así como concentraciones de Hcy marginalmente más altas (10.5 vs. $10.2 \mathrm{mM}$, respectivamente; $p=0.026)^{36}$. Otro estudio en Egipto (50 casos, 70 controles) también reportó mayor concentración de Hcy, MMA y menor concentración de vitB12 sérica en los casos de DTN que en el grupo de controles ${ }^{37}$.

La deficiencia de vitB12 o folato no solo conduce a una síntesis de ADN alterada, sino a eritropoyesis deteriorada, y causa anemia megaloblástica. Aunque representa un pequeño porcentaje de casos de anemia, la anemia megaloblástica es una consecuencia potencial de la deficiencia de folato y vitB12, sin embargo, si se descarta esta última, se usa como un indicador de la gravedad de la deficiencia de folato, mientras que el folato sérico alto es indicativo de deficiencia severa de vitB12. Es importante descartar la deficiencia de vitB12 como causa de síntomas neuropsiquiátricos, ya que no mejorarán con la terapia con ácido fólico. El diagnóstico rápido de la deficiencia de vitB12 es esencial para que el tratamiento sea efectivo, ya que la reversibilidad de los síntomas neurológicos depende en gran medida de esto ${ }^{24,38,39}$.

El estado materno de folato y de vitB12 también se ha asociado con otros resultados infantiles. En un metaanálisis de 18 estudios de cohorte (11,216 observaciones) se reportó que la deficiencia de vitB12 (< 148 pmol/l) se asoció con un aumento del $15 \%$ en el riesgo de un recién nacido con bajo peso al nacer (BPN) (riesgo relativo [RR]: 1.15; IC 95\%: 1.01-1.31) y una probable disminución en el parto pretérmino (RR: 1.21; IC 95\%: 0.99-1.49). Se observó una relación lineal entre las concentraciones de vitB12 y el peso al nacer solo en estudios de países de bajo y medio nivel socioeconómico (particularmente realizados en la India), en donde un aumento en las concentraciones de vitB12 (en una desviación estándar) aumentó el peso al nacer (RR: 0.08; IC 95\%: 0.03-0.14; $I^{2}$ : 0\%). Un aumento en las concentraciones de vitB12 (en una desviación estándar) se asoció con la reducción en el riesgo de parto pretérmino (RR: 0.89; IC 95\%: 0.82$\left.0.97 ; 1^{2}: 0 \%\right)^{40}$.

En una cohorte multiétnica de 12,373 mujeres embarazadas, las concentraciones maternas de folato en etapas tempranas del embarazo se asociaron con un IMC ligeramente mayor en los niños (disminución en 10 unidades $\beta$ : $0.07 \mathrm{~kg} / \mathrm{m}^{2}$; IC 95\%: 0.01-0.13). En este mismo estudio, las concentraciones bajas de vitB12 se asociaron con frecuencias cardiacas mayores (disminución en 10 unidades $\beta$ : 0.49 latidos/min; IC 95\%: 0.11-0.87)

La cohorte prospectiva NEST (Newborn Epigenetics Study; $n=1,304$ mujeres) mostró que los niños de mujeres con las mayores concentraciones de vitB12 experimentaron una ganancia de peso más baja entre el nacimiento y los primeros 3 años de vida, en comparación con los niños de mujeres con las concentraciones más bajas $(\beta=-2203.03, \mathrm{SE}=722.49$, $p=0.003)^{42}$.

En una revisión sistemática de 16 estudios observacionales, se evaluó la asociación entre concentraciones plasmáticas maternas de vitB12 y variables cognitivas ( $n=4$ estudios, 2 en la India), encontrando en dos de estos estudios un mejor desempeño cognitivo en los niños cuyas madres tuvieron concentraciones más altas de vitB12 ${ }^{43}$. Uno de estos estudios, el estudio Pune de Nutrición Materna, observó que niños de 9 años de edad, cuyas madres tuvieron concentraciones plasmáticas de vitB12 > 224 pM, tuvieron un mejor desempeño en tareas de memoria a corto plazo y en atención sostenida, en comparación con niños cuyas madres tuvieron concentraciones mucho más bajas $(<77.0 \mathrm{pM})^{44}$. El otro estudio con resultados positivos, también realizado en la India, reportó que concentraciones plasmáticas de vitB12 maternas > $150 \mathrm{pmol} / \mathrm{l}$ (semana 28) se asociaron significativamente con mayores cocientes de desarrollo (101 vs. $98 ; p=0.035$ ) y cocientes de desarrollo social (93 vs. 91; $p=0.029$ ) en los niños a los 2 años $^{45}$. En los otros dos estudios de cohorte (Canadá e India) no se observaron asociaciones consistentes entre el estado materno de vitB12 y el desarrollo cognitivo en niños de 10 años de edad ${ }^{43}$.

En el estudio Pune de Nutrición Materna (cohorte en la India; 653 binomios madre-hijo) se encontró que los nivel más bajos de vitB12 (a las 18 semanas) y las concentraciones más altas de folato eritrocitario (a las 28 semanas) se asociaron con una mayor resistencia a la insulina en los hijos a los 6 años (B12 < 103 pmol/l: 0.78 [0.42-1.34 HOMA-R vs. B12 $\geq 175$ pmol/l: 0.61 
[0.35-1.00] HOMA-R, $\mathrm{p}=0.04$; folato $<734 \mathrm{nmol} / \mathrm{l}$ : 0.52 [0.28-0.81] HOMA-R vs. folato $\geq 1,269 \mathrm{nmol} / \mathrm{l}$ : 0.85 [0.51-1.27, $\mathrm{p}<0.001)$. Los descendientes de madres con una combinación de altas concentraciones de folato y bajas concentraciones de vitB12 fueron los que presentaron mayor riesgo de resistencia a la insulina. Las mayores concentraciones de folato eritrocitario maternos (a las 28 semanas) se asociaron con mayor adiposidad en los hijos a los 6 años (folato $<734 \mathrm{nmol} / \mathrm{l}: 3.0 \pm 1.0 \mathrm{~kg}$ masa grasa vs. folato $\geq 1,269 \mathrm{nmol} / \mathrm{l}: 3.4 \pm 1.1 \mathrm{~kg}$ masa grasa; $p=0.01)^{33}$.

Por otro lado, también se han reportado efectos adversos asociados a una alta concentración de Hcy. La hiperhomocisteinemia es un factor de riesgo para daño endotelial y enfermedad vascular. Las concentraciones de Hcy dependen del estado de varias vitaminas del complejo $\mathrm{B}$, y se asocian con las características geográficas, culturales y sociales de diferentes poblaciones ${ }^{46}$. En un metaanálisis reciente (2018) se evaluaron estudios que asociaron las concentraciones tempranas de Hcy y el riesgo de desarrollar preeclampsia $(n=4)$, de una manera prospectiva. Dos de estos estudios observaron una asociación de concentraciones mayores de Hcy en el primer trimestre del embarazo en mujeres que después desarrollaron preeclampsia, en comparación con las concentraciones de mujeres que no desarrollaron la enfermedad $(p<0.001)$. Los otros dos estudios no mostraron diferencias. En los estudios de casos y controles (prospectivos), de los 11 estudios incluidos en la revisión, los 11 reportaron que las concentraciones de Hcy promedio fueron significativamente más altas en mujeres que tuvieron preeclampsia en comparación con las que no la presentaron $(p<0.001)^{47}$. Uno de estos estudios fue realizado en México. En este estudio de diseño longitudinal ( $n=252$ mujeres) se reportó un mayor riesgo de preeclampsia en mujeres con altas concentraciones de Hcy (Razón de momios [RM]: 3.933; IC 95\%: 1.523-10.155) ${ }^{48}$. También se observó una asociación entre concentraciones elevadas de Hcy y desprendimiento de placenta ${ }^{47}$.

En otro metaanálisis de 19 estudios (2012) ( $n=$ 21,326 mujeres) se encontró un $25 \%$ de mayor riesgo de tener un recién nacido pequeño para la edad gestacional (PEG) en mujeres con concentraciones elevadas de Hcy (> 90 percentil) (RM: 1.25; IC 95\%: 1.09-1.44) ${ }^{49}$.

Blanco, et al. realizaron un metaanálisis (12 estudios) que mostró que las madres de casos de hendiduras orofaciales no sindrómicas mostraron valores de Hcy en plasma significativamente más altos que las madres de niños sin dicho defecto (Cohen's d: 0.37; IC 95\%: 0.04-0.70; $p=0.026$; 9 estudios) ${ }^{50}$.

En el Estudio Generación R, que incluyó 5,805 binomios madre-hijo, y en donde las muestras se tomaron antes de la semana 18 de gestación, se reportó en promedio un peso al nacer $110 \mathrm{~g}$ menor en recién nacidos de madres en el quintil más alto de Hcy ( $\geq 8.3 \mathrm{mmol} / \mathrm{l})$ en comparación con los recién nacidos de madres con concentraciones en el quintil más bajo ( $\leq 5.8 \mathrm{mmol} / \mathrm{l})$. De la misma forma, fue mayor el riesgo de tener un hijo PEG en las mujeres con concentraciones de Hcy en el quintil más alto (RM: 1.68; $p=0.006$ ) en comparación con las mujeres en el quintil más bajo; y para aquellas en el quintil más bajo de folato $(\leq 9.2 \mathrm{nmol} / \mathrm{l})(\mathrm{RM}: 1.9 ; \mathrm{p}=0.002)$ que en comparación con el más alto ( $\geq 25.9 \mathrm{nmol} / \mathrm{l})$. No se observó un mayor riesgo de parto pretérmino o de preeclampsia en mujeres con altas concentraciones de Hcy (quintil más alto), pero sí se observó el doble de riesgo en mujeres con bajas concentraciones de folato (quintil más bajo) en comparación con el quintil más alto de folato (prematurez RM: 2.17, $p=0.002$; preeclampsia RM: $2.10, p=0.04)^{51}$.

Más recientemente, un estudio multicéntrico, retrospectivo, de casos y controles $(n=563$ embarazadas con resultados adversos [preeclampsia, parto prematuro, BPN, muerte fetal]; $n=600$ controles) encontró que en comparación con los niveles de Hcy del cuartil inferior, los niveles de Hcy del cuartil superior se asociaron con preeclampsia (RM: 2.55; IC 95\%: 1.763.69; $p<0.001$ ), parto prematuro (RM: 3.54 ; IC 95\%: $2.26-5.54 ; p<0.001)$ y BPN (RM: 2.54 ; IC $95 \%$ : $1.62-$ $3.98 ; p<0.001)$. Por el contrario, las concentraciones de folato en el cuartil inferior también se asociaron con preeclampsia (RM: 0.44; IC 95\%: 0.30-0.64; $\mathrm{p}<0.001$ ), parto prematuro (RM: 0.44; IC 95\%: 0.38$0.70 ; p<0.001)$ y BPN (RM: 0.43; IC 95\%: 0.26-0.71; $p=0.001)$ en comparación con las concentraciones de folato en el cuartil superior. Sin embargo, en comparación con los niveles de vitB12 del cuartil superior, las concentraciones de vitB12 del cuartil inferior se asociaron solo con el parto prematuro (RM: 0.56; IC 95\%: 0.37-0.86; $p=0.008$ ). No se observó asociación entre las concentraciones de Hcy, folato y vitB12 para la muerte fetal. Después de ajustar por variables metabólicas y clínicas, la Hcy y el folato se asociaron independientemente con resultados adversos del embarazo $(p<0.05)$; donde la Hcy reveló el riesgo más alto en todas las variables de riesgo (cuartil IV vs. I; RM: 5.89; IC 95\%: 4.08-8.51) y el folato mostró 
el riesgo más bajo en todas las variables protectoras (cuartil IV vs. I; RM: 0.35; IC 95\%: 0.25-0.50) ${ }^{52}$.

En cuanto a desenlaces de neurodesarrollo, los niños de mujeres con concentraciones séricas elevadas de Hcy en el segundo y tercer trimestre del embarazo mostraron menores puntajes en lenguaje expresivo $(\beta:-0.18, p=0.03$ y $\beta:-0.19, p=0.02$, respectivamente), y dominios de motricidad gruesa ( $\beta$ : $-0.23, p=0.008$ y $\beta:-0.30, p=0.001$, respectivamente) de la prueba BSID-III (Bayley Scales of Infant Development), ajustado por confusores ${ }^{53}$.

\section{Suplementación de acido fólico y vitamina B12 en el embarazo}

Es muy importante mantener un equilibrio en la metilación del ADN durante el embarazo. La hipometilación o hipermetilación del ADN, ya sea por exceso o deficiencia de folato, podría estar asociada con diferentes enfermedades neonatales e infantiles. Una metilación aberrante puede promover o inhibir la progresión a algunas enfermedades infantiles, por lo que las dosis apropiadas de ácido fólico son vitales para la salud infantil ${ }^{54}$.

Los estudios de intervención para evaluar la suplementación de folato en humanos y su efecto en la metilación son escasos, poco concluyentes, y pocos han podido relacionarse con desenlaces clínicos. Por otro lado, la suplementación materna con ácido fólico ha mostrado efectos beneficiosos en el peso al nacer, en la prevención de DTN, BPN, PEG, y probablemente en la prevención de preeclampsia y prematurez $z^{17,55-57}$.

En cuanto a DTN y desórdenes congénitos de crecimiento, se han reportado que los cambios en la metilación de IGF2, H19 (asociado a proliferación y diferenciación celular) y GNAS podrían aumentar la susceptibilidad a desarrollarlos ${ }^{54}$.

Datos provenientes de la cohorte multiétnica NEST mostraron que en comparación con los infantes nacidos de madres sin suplementación de ácido fólico antes del embarazo (metilación media: $61.2 \pm 8.1 \%$ ), los niveles de metilación de ADN fueron más bajos entre los infantes nacidos de madres con una suplementación moderada (dentro de la recomendación) (59 $\pm 7.8 \%)$ o suplementación alta (por arriba del límite tolerable de consumo, > 1,000 mcg/d) (media: $58.8 \pm 6.4 \%$ ). Después de ajustar por diferentes variables maternas se observó un menor riesgo de hipermetilación en H19 DMR, con el aumento de la suplementación materna de ácido fólico, tanto para suplementación moderada (RM: 0.47; IC 95\%:
0.22-0.91) como para la alta (RM: 0.31; IC 95\%: $0.11-0.67)^{58}$.

Un estudio de casos $(n=39)$ y controles $(n=49)$ en China encontró niveles de metilación más altos en los sitios 7.8, 9 y 17.18 de H19 DMR ( $p=0.030,0.016$ y 0.050 , respectivamente) en infantes PEG en comparación con aquellos adecuados para la edad gestacional. La asociación fue más fuerte en los infantes de sexo masculino cuyas madres tomaron ácido fólico periconcepcional, en seis sitios H19 $(p<0.05)$. Los autores concluyen que el aumento en los niveles de metilación del ADN en H19 DMR puede estar asociado con ser PEG y la asociación puede ser dependiente del ácido fólico y específica del sexo ${ }^{59}$.

Un ensayo controlado aleatorizado (ECA) doble ciego realizado en Irlanda (FASSTT, Folic Acid Supplementation in the Second and Third Trimesters; $\mathrm{n}=86$ ) encontró que los recién nacidos de madres que recibieron ácido fólico $(400 \mathrm{mcg} / \mathrm{d})$ durante el segundo y tercer trimestre, en comparación con una exposición del suplemento solo en el primer trimestre, tuvieron menor metilación global (LINE-1: $56.3 \pm 1.7 \%$ vs. $57.2 \pm 2.1 \% ; p=0.024$ ), así como de genes específicos relacionados con crecimiento (IGF2: $48.9 \pm$ $4.4 \%$ vs. $51.2 \pm 5.1 \% ; p=0.021$ ) y desarrollo cerebral (factor neurotrófico derivado del cerebro: $2.7 \pm 0.7 \%$ vs. $3.1 \pm 0.8 \% ; p=0.003)^{60}$.

El análisis llevado a cabo por Richmond, et al. muestra evidencia de que el uso materno de suplementos de ácido fólico se asocia con cambios en la metilación del ADN de la descendencia que persisten durante muchos años (edad adulta) después de la exposición en el útero. En este análisis se encontró una menor metilación en cg09112514 (0.8\%; IC 95\%: 0.4-1.2; p = $\left.4.03 \times 10^{-9}\right)$ en los adultos hijos de madres suplementadas en comparación contra los adultos hijos de madres que recibieron placebo. Además, se identificó una reducción de la dosis-respuesta en la metilación en este sitio en relación con la suplementación. En el enfoque regional se identificaron 46 regiones del genoma que se metilaron diferencialmente en respuesta a la suplementación ( $p$ de Sidak $<0.05$ ), incluyendo HLA-DPB2, HLA-DPB1, PAX8 y VTRNA2-161.

\section{Conclusiones}

Un ambiente intrauterino óptimo es fundamental para el establecimiento de patrones epigenéticos embrionarios y de desarrollo fetal que son clave para la salud perinatal y la salud a largo plazo. La susceptibilidad de la metilación del ADN al ambiente 
nutricional es un elemento crítico que puede afectar el desarrollo temprano, influir en los fenotipos y la predisposición al desarrollo futuro de enfermedades.

Las prácticas dietéticas durante el embarazo, particularmente el consumo y la suplementación de micronutrimentos como folato y vitB12 alteran el metabolismo de $1-C$ y pueden afectar las modificaciones de metilación relacionada con un mayor riesgo de resultados perinatales negativos como DTN, prematurez, BPN, preeclampsia, obesidad y resistencia a la insulina materna e infantil, y disminución del desarroIlo neurocognitivo infantil.

Desafortunadamente, la deficiencia de estas vitaminas moduladoras del epigenoma es frecuente a lo largo de los trimestres del embarazo. Por ello debe enfatizarse la importancia del consumo adecuado de alimentos, en particular aquellos ricos en folato y vitB12, y promover la adopción de buenos hábitos de alimentación y estilo de vida para mejorar la salud maternofetal. También parece relevante evaluar y vigilar el estado de estas vitaminas durante el embarazo, para tomar decisiones respecto a la suplementación.

Es necesario mayor investigación sobre dichos nutrimentos y su influencia en el epigenoma para esclarecer los mecanismos metabólicos y reconsiderar y determinar los requerimientos y las dosis óptimos para satisfacer las necesidades que impacten positivamente en el metabolismo del binomio madre-hijo. La evidencia en este respecto será crucial para lograr establecer recomendaciones nutricionales y de suplementación (con dosis e intervalo de tiempo específicos) basadas en perfiles epigenéticos individuales.

\section{Financiamiento}

La presente investigación no ha recibido ninguna beca específica de agencias de los sectores público, comercial, o sin ánimos de lucro.

\section{Conflicto de intereses}

Ameyalli M. Rodríguez-Cano, Otilia Perichart-Perera y Pedro Gutiérrez-Castrellón son consultores del Instituto de Nutrición Nestlé (México). No existe ningún conflicto de interés relacionado a lo expuesto en este artículo.

\section{Responsabilidades éticas}

Protección de personas y animales. Los autores declaran que para esta investigación no se han realizado experimentos en seres humanos ni en animales.
Confidencialidad de los datos. Los autores declaran que en este artículo no aparecen datos de pacientes.

Derecho a la privacidad y consentimiento informado. Los autores declaran que en este artículo no aparecen datos de pacientes.

\section{Bibliografía}

1. Chango A, Pogribny PI. Considering maternal dietary modulators for epigenetic regulation and programming of the fetal epigenome. Nutrients. 2015;7(4):2748-70.

2. Anderson OS, Sant KE, Dolinoy DC. Nutrition and epigenetics: an interplay of dietary methyl donors, one-carbon metabolism and DNA methylation. J Nutr Biochem. 2012;23(8):853-9.

3. Chmurzynska A. Fetal programming: link between early nutrition, DNA methylation, and complex diseases. Nutr Rev. 2010;68(2):87-98.

4. Friso S, Udali S, De Santis D, Choi S-W. One-carbon metabolism and epigenetics. Mol Aspects Med. 2017;54:28-36.

5. Fukuoka H, Kubota T. One-carbon metabolism and lipid metabolism in DOHaD. En: Kubota T, Fukuoka H, editores. Developmental Origins of Health and Disease (DOHaD): From biological basis to clinical significance advances in experimental medicine and biology 1012. Singapore: Springer Singapore; 2018. pp. 3-9.

6. Bianco-Miotto T, Craig JM, Gasser YP, van Dijk SJ, Ozanne SE. Epigenetics and DOHaD: from basics to birth and beyond. J Dev Orig Health Dis. 2017;8(5):513-9.

7. McGee M, Bainbridge S, Fontaine-Bisson B. A crucial role for maternal dietary methyl donor intake in epigenetic programming and fetal growth outcomes. Nutr Rev. 2018;76(6):469-78.

8. Dominguez-Salas P, Cox SE, Prentice AM, Hennig BJ, Moore SE. Maternal nutritional status, $\mathrm{C}(1)$ metabolism and offspring DNA methylation: a review of current evidence in human subjects. Proc Nutr Soc. 2012;71(1):154-65.

9. Kitsiou-Tzeli S, Tzetis M. Maternal epigenetics and fetal and neonatal growth. Curr Opin Endocrinol Diabetes Obes. 2017;24(1):43-6.

10. Hanson MA, Bardsley A, De-Regil LM, Moore SE, Oken E, Poston L, et al. The International Federation of Gynecology and Obstetrics (FIGO) recommendations on adolescent, preconception, and maternal nutrition: "Think Nutrition First." Int J Gynecol Obstet. 2015;131(Suppl 4):S213-53.

11. Vanhees K, Vonhögen IGC, van Schooten FJ, Godschalk RWL. You are what you eat, and so are your children: the impact of micronutrients on the epigenetic programming of offspring. Cell Mol Life Sci. 2014;71(2):271-85.

12. Goyal D, Limesand SW, Goyal R. Epigenetic responses and the developmental origins of health and disease. J Endocrinol. 2019;242(1):T105-19.

13. Kalhan SC. One carbon metabolism in pregnancy: Impact on maternal, fetal and neonatal health. Mol Cell Endocrinol. 2016;435:48-60.

14. McKee SE, Reyes TM. Effect of supplementation with methyl-donor nutrients on neurodevelopment and cognition: considerations for future research. Nutr Rev. 2018;76(7):497-511.

15. Lee H-S. Impact of maternal diet on the epigenome during in utero life and the developmental programming of diseases in childhood and adulthood. Nutrients. 2015;7(11):9492-507.

16. Yajnik CS, Deshmukh US. Fetal programming: Maternal nutrition and role of one-carbon metabolism. Rev Endocr Metab Disord. 2012;13(2):121-7.

17. De-Regil LM, Peña-Rosas JP, Fernández-Gaxiola AC, Rayco-Solon P. Effects and safety of periconceptional fotale supplementation for preventing birth defects. Cochrame Database Syst Rev. 2015;(12):CD007950.

18. Institute of Medicine. Dietary reference intakes for thiamin, riboflavin, niacin, vitamin B6, folate, vitamin B12, pantothenic acid, biotin, and choline. Washington DC: National Academy Press; 1998.

19. Bourges H, Casanueva E, Rosado J. Recomendaciones de ingestión de nutrimentos para la población mexicana. Bases Fisiológicas. Tomo 2. Tomo 2. Energía, proteínas, lípidos. hidratos de carbono y fibra. Panamericana; 2008.

20. Report of a joint FAO/WHO expert consultation. Human vitamin and mineral requirements. Bangkok, Thailand [Internet]. Roma: World Health Organization Food and Agriculture Organization of the United Nations; 2001. Disponible en: http://www.fao.org/3/y2809e/y2809e00.htm

21. Simpson JL, Bailey LB, Pietrzik K, Shane B, Holzgreve W. Micronutrients and women of reproductive potential: required dietary intake and consequences of dietary deficiency or excess. Part I - Folate, Vitamin B12, Vitamin B6. J Matern Neonatal Med. 2010;23(12):1323-43.

22. La Merrill M, Torres-Sánchez L, Ruiz-Ramos R, López-Carrillo L, Cebrián ME, Chen J. The association between first trimester micronutrient intake, MTHFR genotypes, and global DNA methylation in pregnant women. $J$ Matern Neonatal Med. 2012;25(2):133-7.

23. Shane B. Folate status assessment history: implications for measurement of biomarkers in NHANES. Am J Clin Nutr. 2011;94(1):337S-342S. 
24. Finkelstein JL, Layden AJ, Stover PJ. Vitamin B-12 and perinatal health. Adv Nutr. 2015;6(5):552-63.

25. Smith AD, Warren MJ, Refsum H. Vitamin B12. En: Eskin MNA, editor. New research and developments of water-soluble vitamins, Volume 83. Academic Press; 2018. pp. 215-279.

26. Obeid R, Murphy M, Solé-Navais P, Yajnik C. Cobalamin status from pregnancy to early childhood: Lessons from global experience. Adv Nutr. 2017:8(6):971-9.

27. Sukumar N, Saravanan P. Investigating vitamin B12 deficiency. BMJ. 2019;365:I1865.

28. Sukumar N, Rafnsson SB, Kandala N-B, Bhopal R, Yajnik CS, Saravanan P. Prevalence of vitamin B-12 insufficiency during pregnancy and its effect on offspring birth weight: a systematic review and meta-analysis. Am J Clin Nutr. 2016;103(3):1232-51.

29. Allen LH. Folate and vitamin B 12 status in the Americas. Nutr Rev. 2004;62(suppl_1):S29-33.

30. Joubert BR, den Dekker HT, Felix JF, Bohlin J, Ligthart S, Beckett E, et al Maternal plasma folate impacts differential DNA methylation in an epigenome-wide meta-analysis of newborns. Nat Commun. 2016;7(1):10577.

31. Plumptre L, Tammen SA, Sohn K-J, Masih SP, Visentin CE, Aufreiter S, et al. Maternal and cord blood folate concentrations are inversely associated with fetal DNA hydroxymethylation, but not DNA methylation, in a cohort of pregnant Canadian women. J Nutr. 2019;150(2):202-11.

32. Pauwels S, Ghosh M, Duca RC, Bekaert B, Freson K, Huybrechts I, et al. Maternal intake of methyl-group donors affects DNA methylation of metabolic genes in infants. Clin Epigenetics. 2017:9(1):16.

33. Yajnik CS, Deshpande SS, Jackson AA, Refsum H, Rao S, Fisher DJ, et al. Vitamin B12 and folate concentrations during pregnancy and insulin resistance in the offspring: the Pune Maternal Nutrition Study. Diabetologia. 2008;51(1):29-38.

34. Knight BA, Shields BM, Brook A, Hill A, Bhat DS, Hattersley AT, et al. Lower circulating B12 is associated with higher obesity and insulin resistance during pregnancy in a non-diabetic white British population. PLoS One. 2015;10(8):e0135268.

35. Krishnaveni GV, Hill JC, Veena SR, Bhat DS, Wills AK, Karat CLS, et al. Low plasma vitamin B12 in pregnancy is associated with gestational ‘diabesity' and later diabetes. Diabetologia. 2009;52(11):2350-8.

36. Godbole K, Gayathri P, Ghule S, Sasirekha BV, Kanitkar-Damle A, Memane N, et al. Maternal one-carbon metabolism, MTHFR and TCN2 genotypes and neural tube defects in India. Birth Defects Res Part A Clin Mol Teratol. 2011;91(9):848-56.

37. Senousy SM, Farag MK, Gouda AS, El Noury MA, Dabbous OA Gaber KR. Association between biomarkers of vitamin B12 status and the risk of neural tube defects. J Obstet Gynaecol Res. 2018;44(10):1902-8.

38. Green R, Datta Mitra A. Megaloblastic anemias: Nutritional and other causes. Med Clin North Am. 2017:101(2):297-317.

39. Goonewardene M, Shehata M, Hamad A. Anaemia in pregnancy. Best Pract Res Clin Obstet Gynaecol. 2012;26(1):3-24.

40. Rogne T, Tielemans MJ, Chong MFF, Yajnik CS, Krishnaveni G V, Poston $\mathrm{L}$, et al. Associations of maternal vitamin B12 concentration in pregnancy with the risks of preterm birth and low birth weight: A systematic review and meta-analysis of individual participant data. Am J Epidemiol. 2017:185(3):212-23.

41. Krikke GG, Grooten IJ, Vrijkotte TGM, van Eijsden M, Roseboom TJ, Painter RC. Vitamin B12 and folate status in early pregnancy and cardiometabolic risk factors in the offspring at age 5-6 years: findings from the ABCD multi-ethnic birth cohort. BJOG An Int J Obstet Gynaecol. 2016;123(3):384-92

42. McCullough LE, Miller EE, Mendez MA, Murtha AP, Murphy SK, Hoyo C. Maternal B vitamins: effects on offspring weight and DNA methylation at genomically imprinted domains. Clin Epigenetics. 2016;8:8.
43. Venkatramanan S, Armata IE, Strupp BJ, Finkelstein JL. Vitamin B-12 and cognition in children. Adv Nutr. 2016;7(5):879-88.

44. Bhate V, Deshpande S, Bhat D, Joshi N, Ladkat R, Watve S, et al. Vitamin B12 status of pregnant Indian women and cognitive function in their 9-year-old children. Food Nutr Bull. 2008;29(4):249-54.

45. Bhate VK, Joshi SM, Ladkat RS, Deshmukh US, Lubree HG, Katre PA, et al. Vitamin B12 and folate during pregnancy and offspring motor, mental and social development at 2 years of age. J Dev Orig Health Dis. 2012;3(2):123-30.

46. Azzini E, Ruggeri S, Polito A. Homocysteine: Its possible emerging role in at-risk population groups. Int J Mol Sci. 2020;21(4):1421.

47. Gaiday AN, Tussupkaliyev AB, Bermagambetova SK, Zhumagulova SS, Sarsembayeva LK, Dossimbetova MB, et al. Effect of homocysteine on pregnancy: A systematic review. Chem Biol Interact. 2018;293:70-6.

48. López-Alarcón M, Montalvo-Velarde I, Vital-Reyes VS, Hinojosa-Cruz JC, Leaños-Miranda A, Martínez-Basila A. Serial determinations of asymmetric dimethylarginine and homocysteine during pregnancy to predict pre-eclampsia: a longitudinal study. BJOG An Int J Obstet Gynaecol. 2015;122(12):1586-92.

49. Hogeveen M, Blom HJ, den Heijer M. Maternal homocysteine and smaIl-for-gestational-age offspring: systematic review and meta-analysis. Am J Clin Nutr. 2011;95(1):130-6.

50. Blanco R, Colombo A, Pardo R, Suazo J. Maternal biomarkers of methylation status and non-syndromic orofacial cleft risk: a meta-analysis. Int J Oral Maxillofac Surg. 2016;45(11):1323-32.

51. Bergen NE, Jaddoe VWV, Timmermans S, Hofman A, Lindemans J, Russcher $\mathrm{H}$, et al. Homocysteine and folate concentrations in early pregnancy and the risk of adverse pregnancy outcomes: the Generation R Study. BJOG. 2012;119(6):739-51.

52. Liu C, Luo D, Wang Q, Ma Y, Ping L, Wu T, et al. Serum homocysteine and folate concentrations in early pregnancy and subsequent events of adverse pregnancy outcome: the Sichuan Homocysteine study. BMC Pregnancy Childbirth. 2020;20(1):176

53. Thomas S, Thomas T, Bosch RJ, Ramthal A, Bellinger DC, Kurpad AV, et al. Effect of maternal vitamin B12 supplementation on cognitive outcomes in South Indian children: A randomized controlled clinical trial. Matern Child Health J. 2019;23(2):155-63.

54. Liu H-Y, Liu S-M, Zhang Y-Z. Maternal folic acid supplementation mediates offspring health via DNA methylation. Reprod Sci. 2020;27(4):963-76.

55. Jonker H, Capelle N, Lanes A, Wen SW, Walker M, Corsi DJ. Maternal folic acid supplementation and infant birthweight in low- and middle-income countries: A systematic review. Matern Child Nutr. 2020;16(1):e12895.

56. Li B, Zhang X, Peng X, Zhang S, Wang X, Zhu C. Folic acid and risk of preterm birth: A meta-analysis. Front Neurosci. 2019:13:1284

57. Liu C, Liu C, Wang Q, Zhang Z. Supplementation of folic acid in pregnancy and the risk of preeclampsia and gestational hypertension: a meta-analysis. Arch Gynecol Obstet. 2018/07/05. 2018;298(4):697-704.

58. Hoyo C, Murtha AP, Schildkraut JM, Jirtle RL, Demark-Wahnefried W, Forman MR, et al. Methylation variation at IGF2 differentially methylated regions and maternal folic acid use before and during pregnancy. Epigenetics. 2011:6(7):928-36

59. Qian Y-Y, Huang X-L, Liang H, Zhang Z-F, Xu J-H, Chen J-P, et al. Effects of maternal folic acid supplementation on gene methylation and being small for gestational age. J Hum Nutr Diet. 2016;29(5):643-51.

60. Caffrey A, Irwin RE, McNulty H, Strain JJ, Lees-Murdock DJ, McNulty BA, et al. Gene-specific DNA methylation in newborns in response to folic acid supplementation during the second and third trimesters of pregnancy: epigenetic analysis from a randomized controlled trial. Am J Clin Nutr. 2018:107(4):566-75.

61. Richmond RC, Sharp GC, Herbert G, Atkinson C, Taylor C, Bhattacharya $S$, et al. The long-term impact of folic acid in pregnancy on offspring DNA methylation: follow-up of the Aberdeen Folic Acid Supplementation Trial (AFAST). Int J Epidemiol. 2018;47(3):928-37. 